Mélanges

de l'École française de Rome

Moyen Âge

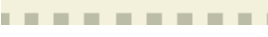

Mélanges de l'École française de Rome Moyen Âge

132-1 | 2020

Bibliothérapies médiévales - Varia - Outils et méthodes pour l'histoire des Églises entre Orient et Occident

\title{
Rappresentazioni del potere tra giudicato d'Arborea, Corona d'Aragona e Regno di Napoli
}

Nicoletta Usai

\section{CpenEdition}

Journals

Edizione digitale

URL: http://journals.openedition.org/mefrm/6872

ISSN: $1724-2150$

Editore

École française de Rome

\section{Edizione cartacea}

Data di pubblicazione: 1 gennaio 2020

ISBN: 978-2-7283-1404-1

ISSN: $1123-9883$

Notizia bibliografica digitale

Nicoletta Usai, «Rappresentazioni del potere tra giudicato d'Arborea, Corona d'Aragona e Regno di Napoli », Mélanges de l'École française de Rome - Moyen Âge [Online], 132-1 | 2020, Messo online il 26 mars 2020, consultato il 06 avril 2020. URL : http://journals.openedition.org/mefrm/6872

Questo documento è stato generato automaticamente il 6 aprile 2020

(c) École française de Rome 


\title{
Rappresentazioni del potere tra giudicato d'Arborea, Corona d'Aragona e Regno di Napoli
}

\author{
Nicoletta Usai
}

1 L'analisi dei fatti storici e della cultura materiale restituisce oggi un quadro del Trecento sardo molto articolato, nel quale esistono varie figure di spicco, il cui carisma è testimoniato non solo dalle fonti storiche, ma anche da manufatti recentemente ricondotti ad una corretta lettura e contestualizzazione. ${ }^{1}$ Il XIV secolo è attraversato in Sardegna dal confronto tra la Corona d'Aragona e il giudicato d'Arborea, entità territoriale che ancora è testimonianza dell'assetto istituzionale dei secoli precedenti. ${ }^{2}$ Nella dialettica si inserisce anche la presenza di famiglie signorili ${ }^{3}$, pisane e genovesi, stanziate in porzioni territoriali del nord e sud dell'isola, che mal sopportano la convivenza con i Catalani ${ }^{4}$.

2 Di particolare interesse, per gli esiti e per le ricadute culturali sul territorio, sono le due corti a Barcellona e a Oristano, nelle quali crescono e si formano coloro che cambiano l'andamento degli eventi. In particolare Pietro, sovrano della Corona con il titolo di Pietro IV il Cerimonioso, e i principi Mariano e Giovanni d'Arborea, figli del giudice Ugone II, sono tra i principali responsabili, in maniera diretta o indiretta, della realizzazione di opere che servono anche a comunicare dei precisi messaggi connessi all'ideologia del potere.

3 Mariano IV, sovrano del giudicato d'Arborea alla metà del secolo, è tra i personaggi che si trovano a vivere un momento cruciale della storia medievale non solo sarda. ${ }^{5}$

4 Figlio di Ugone II, questi salì al trono nel 1347, succedendo al fratello Pietro III. ${ }^{6}$ Alcuni documenti, conservati nell'Arxiu Històric de Protocols de Barcelona e redatti tra il 1336 e il $1362 \mathrm{da}$ alcuni notai catalani ${ }^{7}$, consentono di precisare alcune consuetudini della corte giudicale. Negli atti notarili sono protagonisti i figli di Ugone II, Pietro, Giovanni e Mariano unitamente alla moglie di quest'ultimo, Timbors. Tra le note di spesa inserite in un libro dei conti figurano i costi sostenuti per l'acquisto di carni ed altri cibi per $\mathrm{i}$ banchetti nuziali di Mariano e del fratello Giovanni, entrambi celebrati a Barcellona. 
Anche i preparativi per il rientro nell'isola sono noti ${ }^{8}$. In questi risulta il pagamento di tessuti di lana di differenti colori e altri oggetti di pregio destinati ad essere portati in dono alla famiglia del donnikellu.

5 Nel 1362, quando i rapporti con la Corona d'Aragona sono oramai tesi, si ipotizza che da Barcellona arrivino alcuni oggetti, di proprietà della moglie di Mariano, descritti con cura nell'atto notarile. Tra i beni risultano due saliere, una decorata con un serpente alato, l'altra con un non meglio specificato animale, quattro spade e una cintura, tutte con applicazioni in argento, ventuno sacramentali con impressa l'immagine dell'Agnus Dei in argento, più una serie di altri oggetti dello stesso metallo. Tra questi si individuano una coppa munita di piede, due brocche, dodici scodelle, ma anche quattro elmi, «due bancals di stoffa di lana con le insegne della casa d'Arborea, altri tagli di stoffa e due teli di pelliccia» ${ }^{9}$. È stato ipotizzato che il sovrano Mariano potesse partecipare a tornei cavallereschi, come sembrerebbero indicare le spade e gli elmi. Certamente, afferma Rosanna Lusci «questi oggetti, al pari di quelli di cui si è già detto, rientrano perfettamente tra i beni di cui una famiglia nobile dell'epoca, come quella degli Arborea, poteva disporre» ${ }^{10}$.

6 L'immagine del giovane principe Mariano (fig. 1), rappresentata nel polittico di Ottana (Nuoro), dipinto su tavola datato al secondo quarto del Trecento, resta una delle testimonianze più interessanti del Trecento isolano. ${ }^{11} \mathrm{Il}$ principe ${ }^{12}$ è presentato inginocchiato, con le mani giunte, ai piedi della Madonna in trono con Bambino. L'iscrizione nel basamento del trono, sul quale è seduta Maria con Gesù bambino, definisce Mariano $D[o m i] n[u] s$ Gociani et M[amille], titolo che viene sostituito nel 1338 con quello di Comes de Guciani. ${ }^{13}$ È quindi una datazione ad annum quella che chi scrive ipotizza per il ritratto del futuro sovrano contenuto nel polittico di Ottana, in quel 1338 che poi lo vedrà diventare conte. Una giusta riflessione fatta da Alessandro Soddu sottolinea anche come, se Mariano fosse stato già conte all'epoca della realizzazione del polittico, certamente si sarebbe fatto individuare con la titolatura corrente, e non con una denominazione oramai obsoleta ${ }^{14}$. La presenza di Silvestro, attestato solo dal 1339 alla guida della Diocesi, in posizione simmetrica rispetto a Mariano non costituisce un problema insormontabile per anticipare la datazione del polittico, per tradizione collocata tra il 1339 e il 1344 , al $1338 .^{15}$ 
Fig. 1 - Ottana (Nuoro), polittico con i santi Francesco e Nicola, particolare del donnikellu Mariano (da Serra 1990, p. 65).

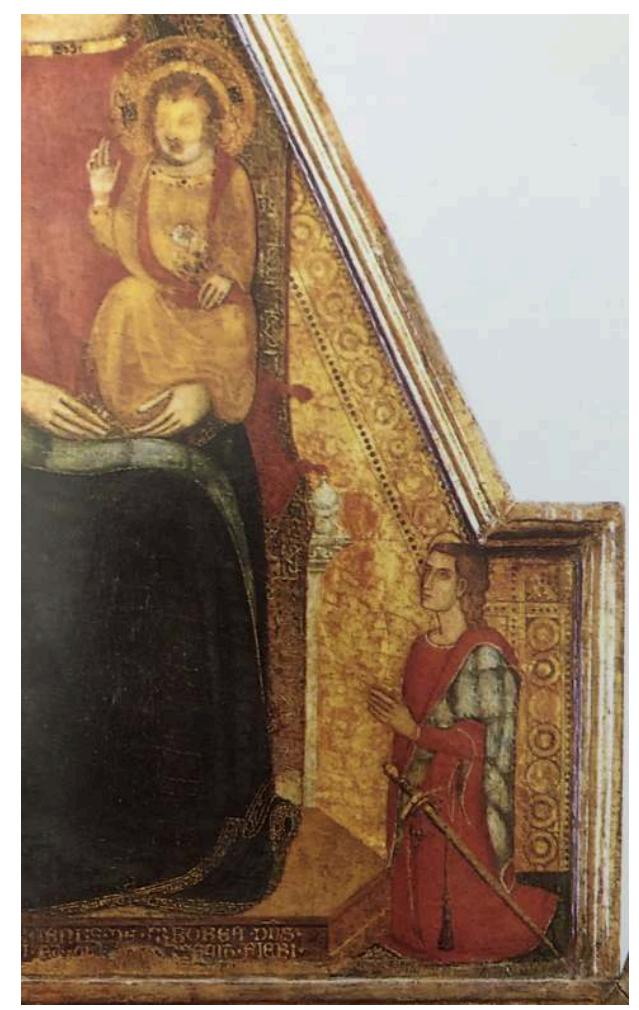

7 È difficile stabilire fino a che punto abbia inciso, nelle specifiche scelte operate nel polittico di Ottana, la volontà del committente laico. Se infatti per il vescovo di Ottana, il francescano Silvestro, si può ipotizzare un ruolo nelle decisioni relative ai contenuti da inserire nel dipinto, per il donnikellu si potrebbe pensare che, perlomeno, abbia indicato come farsi rappresentare nell'opera che, almeno in parte, aveva voluto e finanziato. È quindi ipotizzabile che sia stato Mariano stesso a dare suggerimenti sull'immagine che doveva comparire nel polittico, forse prendendo a modello uno o più esempi visti alla corte barcellonese nei lunghi anni di soggiorno catalano. Volendo portare solo un esempio, che per cronologia affine partecipa dello stesso humus culturale che dà vita alla pala di Ottana, si può confrontare l'immagine isolana con l'effige di Enrico di Trastámara, re di Castiglia e León tra 1369 e 1379, che si fa ritrarre nel dipinto su tavola della Virgen del Tobed ${ }^{16}$, databile agli anni compresi tra il 1359 e il $1362^{17}$. Enrico, non ancora re, è rappresentato ai piedi della Vergine già con il capo coronato, con la moglie Juana Manuel di Castiglia e i due figli. Il futuro sovrano si fa ritrarre già come re, nonostante ancora non lo sia. Rappresentato in ginocchio, con le mani giunte, ha appesa alla vita la spada, attributo immancabile del suo status sociale. Non si intende istituire un confronto formale tra le due opere ma sottolineare come, dal punto di vista culturale e concettuale, il ritratto di Mariano molto si avvicini a questa tipologia di ritrattistica, certamente con finalità encomiastica. ${ }^{18}$

Paola Vitolo sottolinea come, tra XIII e XIV secolo, iniziative dal forte impatto simbolico celebrino il prestigio delle dinastie, la loro forza, la loro santità. La realizzazione delle tombe reali all'interno dell'abbazia di Westminster, a Londra, o a Saint Denis, vicino Parigi, o ancora la costruzione della Sainte chapelle, nella medesima città francese, sono testimoni del legame presente tra le dinastie regnanti e la devozione religiosa: 
souverains et princes trouvèrent des arguments efficaces et des symboles de légitimité, auxquels ils firent surtout appel en temps de crise dynastique et de tensions internes, en les réinterprétant souvent avec beaucoup d'originalité ${ }^{19}$.

9 Per quanto concerne la Corona d'Aragona fino agli anni '30 del XIV secolo non sembra nemmeno progettarsi la costruzione di una tomba reale, probabilmente per mancanza di fondi, già dirottati in campagne belliche di espansione territoriale. Solo con Pietro il Cerimonioso, regnante tra 1336 e 1387, i membri della dinastia, già sepolti, vengono trasportati in un luogo consono al loro status. Il sovrano commissiona e fa realizzare, nell'arco di tre decadi, un pantheon reale nel monastero di Poblet, dove trovano dimora le spoglie di Alfonso il Casto e Giacomo il Conquistatore, predecessori del Cerimonioso e considerati alla stregua di reliquie da tesaurizzare. ${ }^{20}$ Differenti personalità si succedono alla direzione del cantiere. Pere de Guines, Aloi de Montbrai, Jaume Cascalls, Jordi de Déu mantengono un rapporto molto stretto con il monarca che interviene in maniera diretta nelle scelte relative al cantiere ${ }^{21}$. La strategia comunicativa, adottata per veicolare messaggi visivi e di propaganda, prevede la realizzazione di sepolture non terragne ma sopraelevate rispetto al pavimento, con i sepolcri tutti alla stessa altezza, alternati a stemmi reali che dovevano evidenziare i legami tra i vari dinasti. Nel 1377 il ruolo di sepolcro reale di Poblet viene sancito anche dalla donazione, al monastero, delle cronache dei conti di Barcellona e dei re d'Aragona. In questo modo Poblet diventa il centro simbolico della dinastia, specchio del suo glorioso passato e custode della sua memoria storica ${ }^{22}$. Joan Molina Figueras afferma che

ningún monarca mostró mayor interés por la preservación de la gloriosa memoria del linaje como Pedro IV. Dan fe de ello las crónicas que escribió e hizo escribir a sus colaboradores (desde la dedicada a su propio reinado a las que versaban sobre la historia dinástica) así como los múltiples sermones de cortes, cartas y discursos públicos en los que se rememoraban las heroicas gestas de sus antecesores ${ }^{23}$.

Il Cerimonioso dunque è fautore di un'ampia e strutturata operazione di propaganda, volta a glorificare la monarchia aragonese, attraverso l'utilizzo di retorica e magniloquenza. ${ }^{24}$ La preservazione e diffusione della memoria dinastica, intesa come pilastro sul quale fondare questa campagna di celebrazione aulica, diventa quasi un'ossessione, che porta il sovrano a esercitare una stretta forma di controllo rispetto a ogni attività connessa a questo scopo. In quest'ottica si può leggere il coinvolgimento personale di Pietro IV in molti degli scritti dedicati a lui o ai suoi predecessori. ${ }^{25}$ Le lettere di cancelleria e le ordenacions della Casa Reale riportano annotazioni fatte dal sovrano in persona, mentre il processo di redazione di sermoni di corte o discorsi solenni, con evidente intento di propaganda, è seguito passo per passo dal monarca ${ }^{26}$. Sono soprattutto le cerimonie, gli spettacoli regi e le immagini ad essere utilizzate come potente mezzo di celebrazione e trasmissione della storia dinastica ${ }^{27}$. In quest'ottica non stupisce quindi che siano realizzate negli anni centrali del Trecento due straordinarie opere di glorificazione del potere monarchico: il già citato pantheon reale nella chiesa del monastero di Poblet e la serie di effigi di alabastro, degli undici conti e degli otto conti-re di Barcellona, già nel Palacio Reyal..$^{28}$ Solo i documenti riportano notizia delle sculture il cui incarico fu affidato ad Aloi de Montbrai tra $1340 \mathrm{e}$ $1342^{29}$. Nessuna descrizione dettaglia le forme di queste effigi, la cui realizzazione occupò diverse decadi fino almeno al 1360. Tuttavia emerge da alcuni documenti che la loro collocazione definitiva dovesse essere nelle mensole o nei capitelli dell'appena concluso Salón del Tinell ${ }^{30}$, vasta sala all'interno del Palazzo Reale, a pianta rettangolare scandita da archi diaframma. ${ }^{31}$ 
11 I presunti ritratti di alcuni dei giudici della casa d'Arborea, collocati nei presbiteri di due chiese ricomprese nel territorio statale, potrebbero rientrare in queste forme di autorappresentazione e di comunicazione del potere, motivate anche da un momento di crisi politico-istituzionale..$^{32}$ Il più noto di questi rilievi scultorei raffigurerebbe Eleonora d'Arborea (fig. 2), con i lunghi capelli sciolti sulle spalle, individuata in uno dei quattro peducci che sorreggono la volta del presbiterio della chiesa di San Gavino (fig. 3) a San Gavino Monreale (Sud Sardegna). ${ }^{33}$ Questa è ubicata nelle vicinanze del castello residenziale omonimo posto al confine tra il giudicato d'Arborea e l'ex giudicato di Cagliari. Della struttura originaria, ascrivibile alla seconda metà del XIV secolo, si conserva il presbiterio a pianta quadrangolare con volta a crociera costolonata $^{34}$. Proprio i sostegni su cui poggiano i costoloni riprodurrebbero, oltre ad Eleonora, suo marito Brancaleone Doria, suo padre Mariano IV con corona e scettro (fig. 4), Ugone III con la figlia Benedetta. ${ }^{35}$ Analogamente anche nella chiesa monastica di Santa Chiara a Oristano (fig. 5) sarebbero custodite altre rappresentazioni della famiglia regnante. Sempre l'abside ospiterebbe Mariano II, sovrano tra 1250 e 1291, Pietro III e la moglie Costanza di Saluzzo e, nuovamente, Mariano IV. ${ }^{36}$ Queste raffigurazioni antropomorfe sono rese con teste globoidi, incassate in corpi appena accennati dai quali si dipartono grandi mani, sproporzionate rispetto al resto della figura ${ }^{37}$ L'identificazione di questi rilievi scultorei con i membri della casata giudicale troverebbe un conforto e una giustificazione proprio nelle prassi in uso in quegli anni nelle principali corti europee ${ }^{38}$ Come già visto, la Corona catalano-aragonese proprio negli anni centrali del Trecento basa la sua legittimazione sulla celebrazione della storia del lignaggio concretizzata nella trasmissione del potere di generazione in generazione. ${ }^{39}$ Con la commissione della serie di sculture commemorative dei regnanti, già nel Salón del Tinell del Palazzo Reale di Barcellona, il monarca Pietro Il Cerimonioso esemplifica la continuità dinastica, fondamento stesso della legittimità e del prestigio della Corona, attraverso una comunicazione visuale che doveva risultare di grande efficacia e altrettanta chiarezza per chi osservava. ${ }^{40}$ 
Fig. 2 - San Gavino Monreale (Sud Sardegna), San Gavino, peduccio del presbiterio (foto autore).

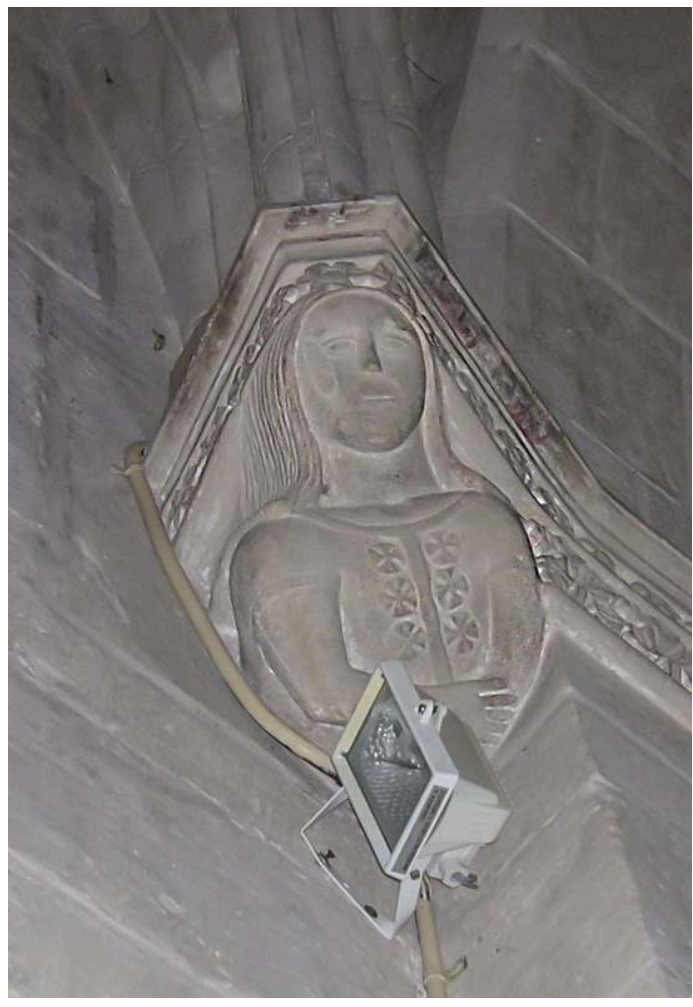

Fig. 3 - San Gavino Monreale (Sud Sardegna), San Gavino, interno (foto autore).

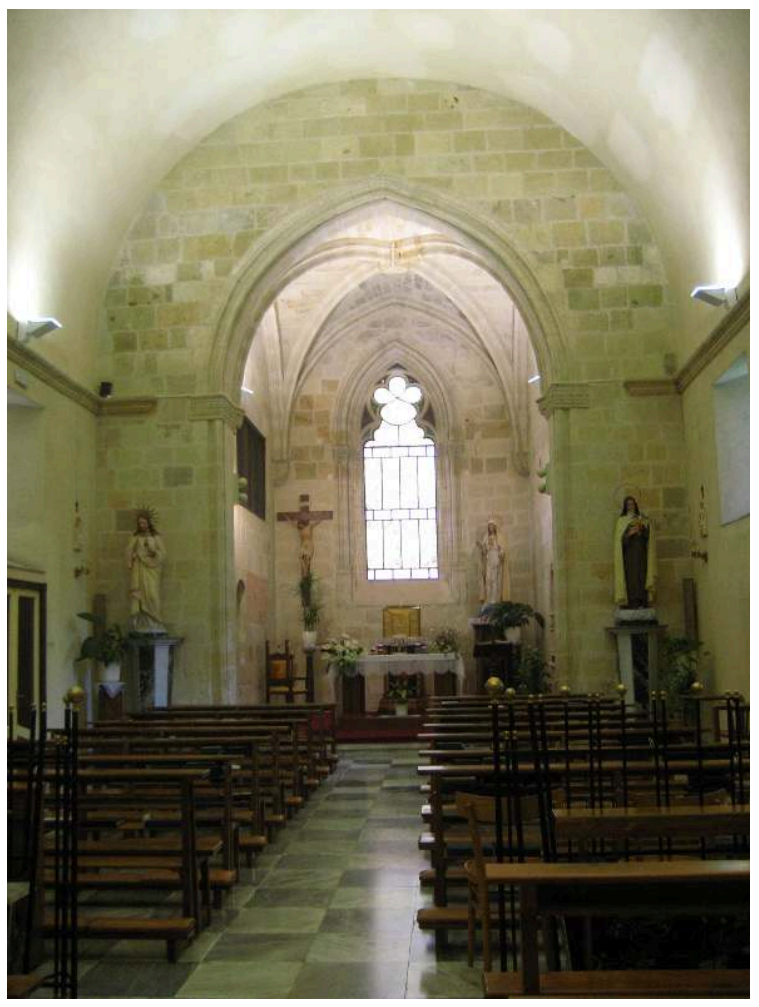


Fig. 4 - San Gavino Monreale (Sud Sardegna), San Gavino, peduccio del presbiterio (foto autore).

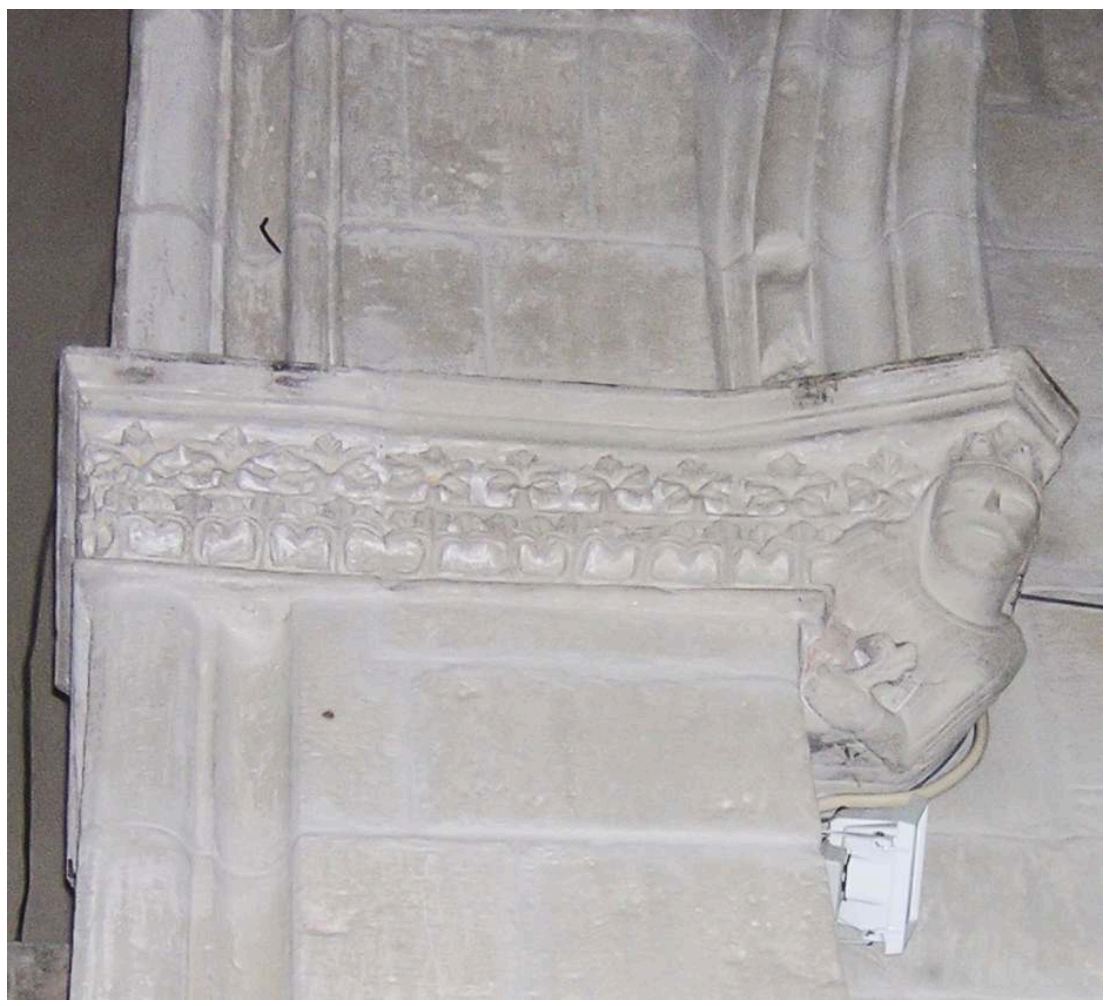

Fig. 5 - Oristano, Santa Chiara, presbiterio (foto autore).

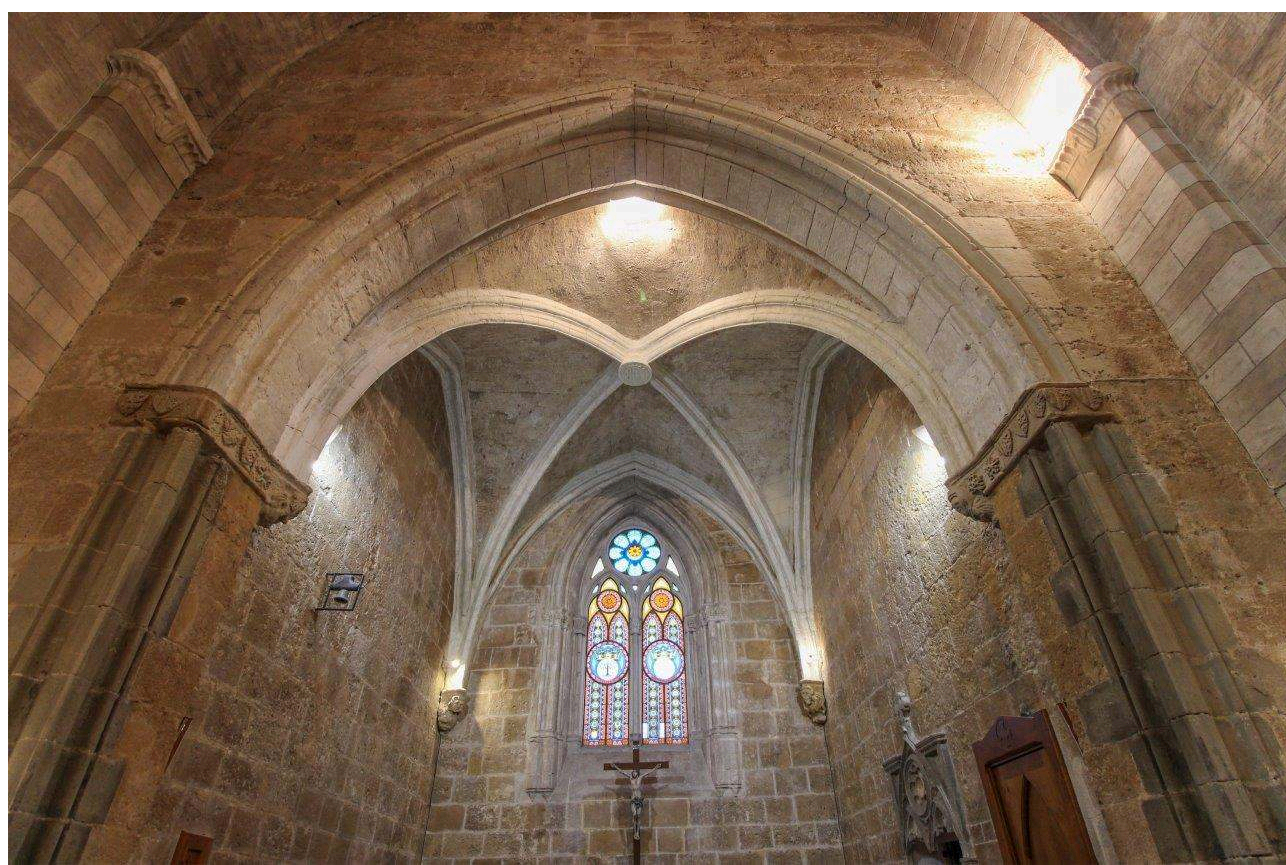

12 Nel caso dei busti isolani si evidenzia come, effettivamente, alcuni dei personaggi esaminati portino sul capo proprio una corona. Oltretutto la chiesa di Santa Chiara a Oristano ospita numerosi stemmi della casata regnante, con albero deradicato e pali d'Aragona. ${ }^{41}$ Oltre al concio in facciata, sovrastante il portale d'ingresso, all'interno della chiesa si trovano dei fregi decorati con gli stemmi dei giudici alternati a motivi 
fitomorfi, collocati sia nell'intradosso sia nella parete esterna dell'arco che immette nel presbiterio (fig. 6). Lo stemma della casa regnante si trova anche nella lastra sepolcrale di Costanza di Saluzzo, già collocata nel chiostro del convento e oggi riposizionata all'interno dell'aula della chiesa. ${ }^{42}$ Gli stemmi sono dipinti anche in alcune delle mensole dell'originaria copertura lignea dell'edificio (fig. 7), oggi ricollocate nella cantoria $^{43}$ (fig. 8). L'ipotesi che i giudici arborensi si facciano rappresentare all'interno di un edificio religioso acquista decisa consistenza soprattutto in virtù del legame, ampiamente attestato dalle fonti documentarie, che gli stessi regnanti isolani avevano negli anni centrali del XIV secolo soprattutto con la chiesa oristanese di Santa Chiara ${ }^{44}$. Chi scrive si è occupato, in diverse sedi, dell'analisi del monumento e dei dipinti in esso contenuti. ${ }^{45}$ Nonostante i problemi legati alla cronologia e alle fasi di vita del complesso, la documentazione relativamente abbondante riconducibile al XIV secolo chiarisce molti aspetti. Una serie di missive del Papa aiuta a dimostrare come nel Trecento la chiesa fosse attiva e frequentata dalla famiglia giudicale: è del 30 giugno 1345 l'atto con il quale il Pontefice concede al giudice Pietro III il permesso di accedere al monastero, precisando come questo includesse la preesistente chiesa di San Vincenzo Martire, della quale negli anni ' 80 del secolo scorso sarebbero state trovate tracce durante lavori di restauro della pavimentazione. ${ }^{46}$ Lascito di Costanza di Saluzzo, moglie del giudice Pietro III, al monastero oristanese fu la villa di Molins del Rey in Catalogna $\mathrm{a}^{47}$. La stessa Costanza, alla morte del consorte, si ritirò nel monastero clariano e là fu sepolta, come testimonia la sua lapide ${ }^{48}$. Di assoluto rilievo fu altresì l'atto promulgato da Mariano IV d'Arborea, nel 1368, con il quale veniva concessa alle Clarisse una donazione perpetua dell'entità di 260 lire, vincolante anche per gli eredi del sovrano, ulteriore testimonianza della devozione della casa regnante e del legame con il complesso monastico. ${ }^{49}$

Fig. 6 - Oristano, Santa Chiara, presbiterio, particolare degli stemmi scolpiti (foto autore).

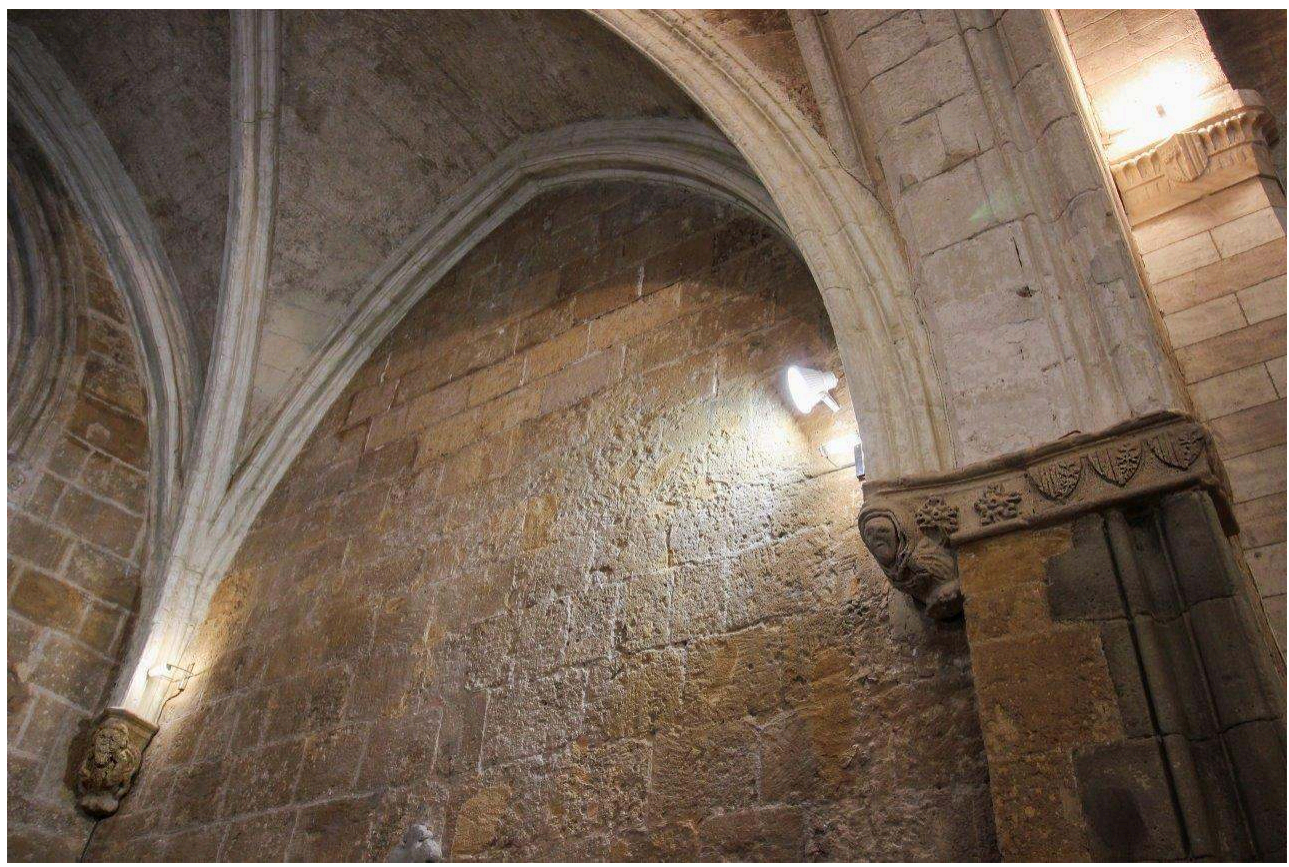


Fig. 7 - Oristano, Santa Chiara, mensola lignea con stemma dipinto (foto autore).

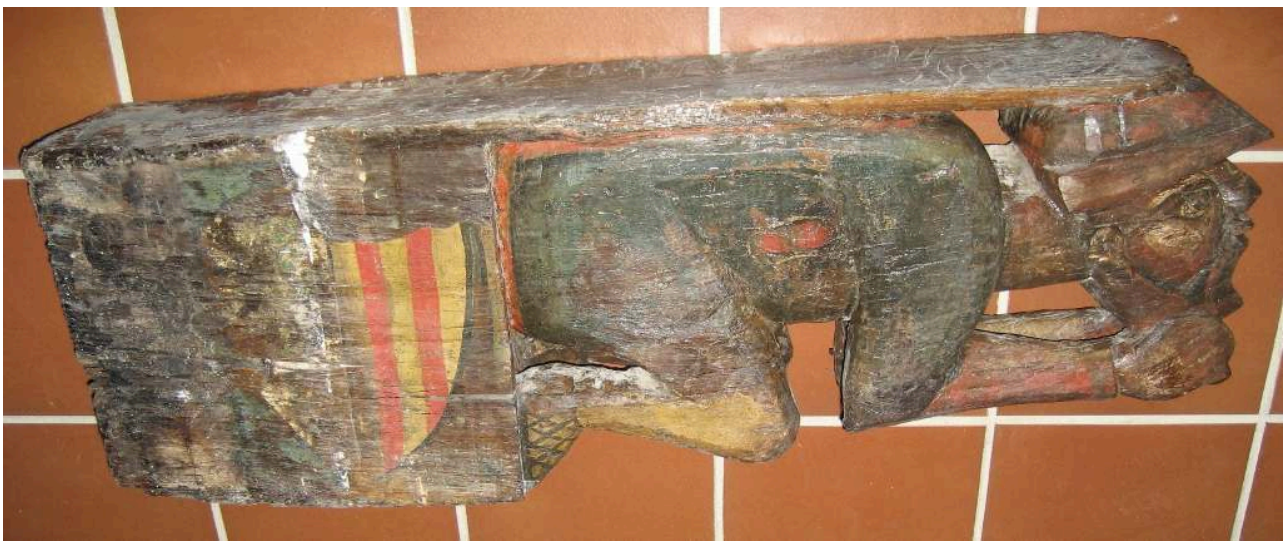

Fig. 8 - Oristano, Santa Chiara, cantoria con mensole lignee (foto autore).

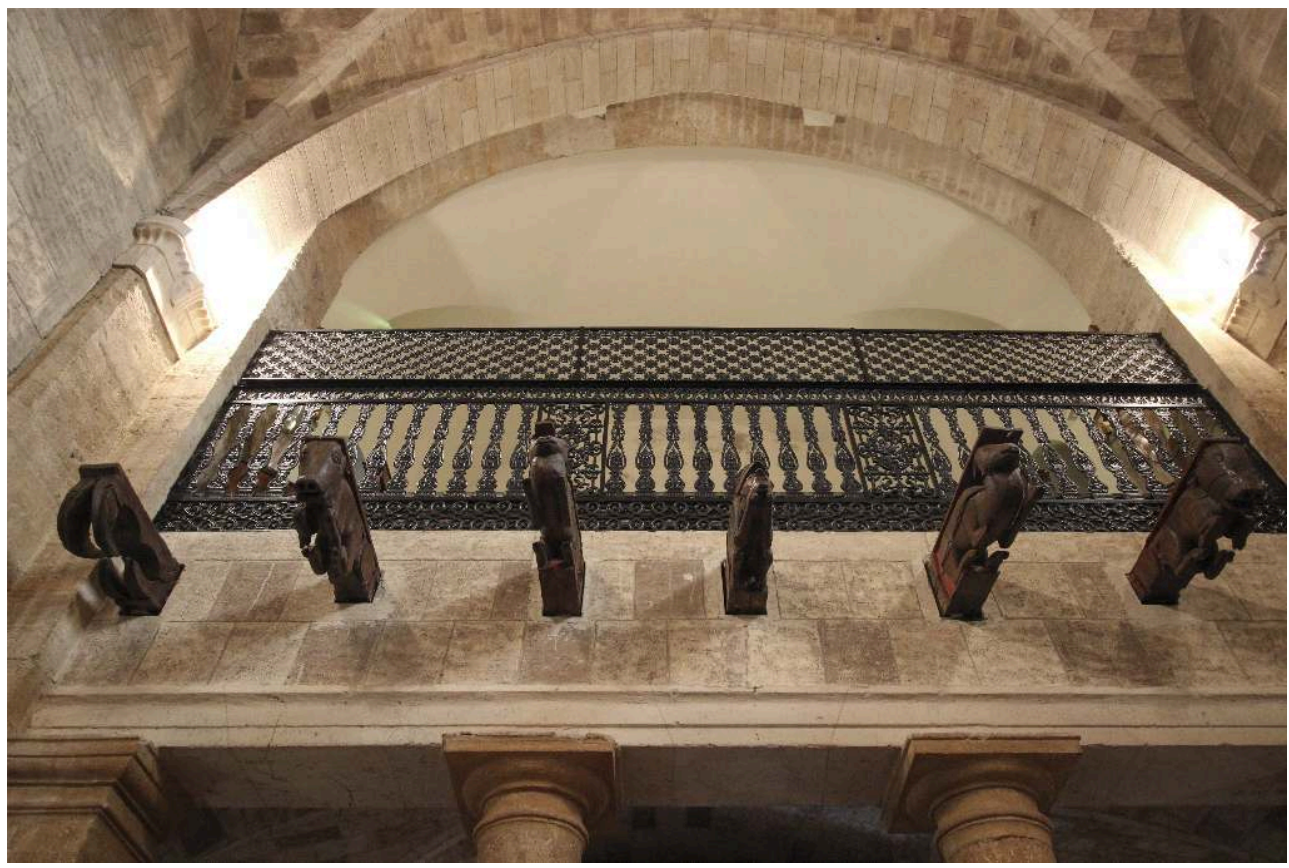

È da notare che anche a Napoli i reali angioini, Roberto e Sancia, contribuirono a finanziare, tra il 1310 e il 1340, la realizzazione della chiesa di Santa Chiara (già del Corpo di Cristo o Ostia Santa), costruita accanto ad un monastero francescano doppio: «Elle fondit dans une perspective unique la célébration de la dynastie angevine et une dévotion sincère et profonde à l'Eucharistie, que la reine Sancia avait héritée de sa tradition familiale et qui était l'un des traits les plus remarquables de sa religiosité» ${ }^{50}$. Eletto a pantheon reale della dinastia, ancora si discute se fosse stato già concepito all'inizio con questa funzione o se le sia stata attribuita in un secondo momento. ${ }^{51}$ Ancora un monastero, ma di monaci cistercensi, il già citato monastero di Poblet, diventa il centro di conservazione e di duratura celebrazione della memoria dei sovrani catalano-aragonesi, per volontà di Pietro il Cerimonioso. ${ }^{52}$

Per quanto riguarda la Sardegna, Mariano IV d'Arborea è il munifico mecenate del complesso monastico di Oristano e non solo. Alla luce degli studi più recenti si capisce meglio quanto forte sia stata la sua azione politica e, ad avviso di chi scrive, altrettanto 
incisivo è stato il suo intervento dal punto di vista culturale. Il legame, pur molto travagliato, con il fratello Giovanni, con il quale ha condiviso l'educazione a Barcellona, genera due opere fondamentali quali il già citato polittico di Ottana e il ciclo pittorico di Nostra Signora de Sos Regnos Altos a Bosa. ${ }^{53}$ La committenza dei dipinti bosani (fig. 9), ascritta alternativamente a uno dei due fratelli, Mariano o Giovanni, si lega strettamente ai fatti che occorsero proprio ai due e che li videro contrapposti, con l'incarcerazione di Giovanni nel 1349 da parte del fratello. Se è vero che Giovanni soggiorna con la consorte barcellonese Sibilla de Montcada a Bosa per circa 10 anni alla stessa Sibilla peraltro è concesso di rimanere nel castello anche dopo l'incarcerazione del marito- è anche accertato che Mariano IV prenda possesso del castello nel 1352 e ne faccia un suo quartier generale fino alla morte. È quindi evidente che la chiesa e il castello furono di estrema rilevanza per entrambi. ${ }^{54}$

Fig. 9 - Bosa (Oristano), Nostra Signora de Sos Regnos Altos, interno (foto autore).

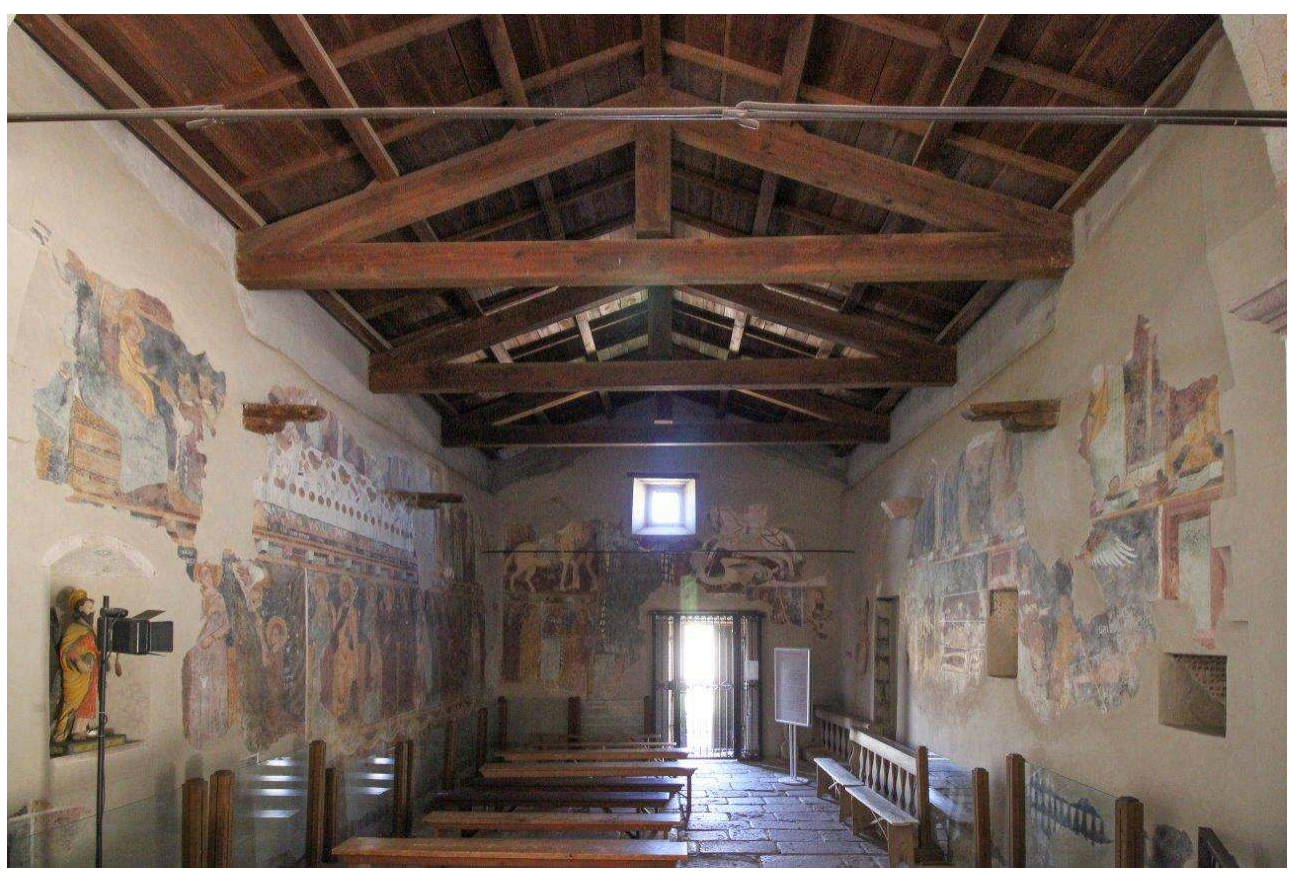

Nell'analisi del ciclo pittorico, oggetto di diversi studi ${ }^{55}$, è da evidenziare il ruolo svolto dall'ideatore/iconografo, che ha operato scelte molto precise rispetto ai temi da trattare. Data la particolare natura delle scelte operate è assai probabile che il committente-concepteur ${ }^{56}$ sia un religioso, mentre a un laico, lo stesso Giovanni d'Arborea, si potrebbe attribuire il ruolo di finanziatore dei lavori. L'inserimento di alcune figure all'interno del ciclo bosano, come san Costantino e sant'Elena collocati nella controfacciata, risulta di problematica spiegazione (fig. 10). Non soddisfa a pieno la spiegazione, invalsa nella critica precedente ${ }^{57}$, che il filo conduttore dei dipinti fosse, in maniera generica, la mistica francescana. Proprio partendo da Costantino ed Elena in controfacciata, che comunque si connotano come un unicum iconografico per questo periodo, chi scrive ha avanzato recentemente una nuova proposta di lettura e interpretazione dell'intero ciclo pittorico che si lega ad una serie di eventi ben precisi. Nel 1342 i Francescani ottengono, grazie a due distinte bolle papali - Gratias agimus e Nuper carissime - la custodia dei Luoghi Santi, grazie all'intervento dei reali di Napoli, Roberto d'Angiò e Sancia di Maiorca. Ricade dunque sotto la tutela dei Minori il Santo Cenacolo, i santuari e le cappelle del Monte Sion, la basilica del Santo Sepolcro. ${ }^{58} \mathrm{La}$ 
custodia dei luoghi della vita e della passione di Cristo si lega in maniera indissolubile anche alla grande venerazione che i seguaci di Francesco tributavano alla Croce di Cristo, poiché nelle loro tradizioni Francesco aveva ricevuto le stimmate proprio il 14 settembre, giorno dell'Esaltazione della Croce $^{59}$. Potrebbe dunque essere questo evento ad aver spinto l'ideatore del ciclo, con tutta probabilità francescano, a celebrare l'Ordine e il legame con i luoghi santi, ponendo all'interno dei dipinti l'Adorazione dei Magi, ambientata a Betlemme, la Coena Domini, ma anche Elena, colei che ha trovato la Croce di Cristo, e Costantino, vera radice del Cristianesimo. La celebrazione dell'evento passa anche attraverso la glorificazione dei sovrani angioini, per mezzo del novello santo Ludovico da Tolosa, fratello di Roberto d'Angiò. È altresì ipotizzabile che il finanziatore del ciclo abbia voluto in qualche modo dire la sua, come sembrerebbe indicare la presenza dei santi Lorenzo e Francesco e, probabilmente, di san Nicola. Cosi si chiamano i fratelli di Giovanni d'Arborea, che proprio in quegli anni accompagnava Pietro il Cerimonioso in guerra. ${ }^{60}$ Sembra dunque possibile ancorare il ciclo di Bosa proprio agli anni ricompresi tra il 1342 e il 1343, in cui Giovanni era il feudatario più potente dell'isola, fedele al Cerimonioso e, come era tradizione di famiglia, estremamente legato ai Francescani. ${ }^{61}$

Fig. 10 - Bosa (Oristano), Nostra Signora de Sos Regnos Altos, interno, particolare dei dipinti in controfacciata (foto autore).

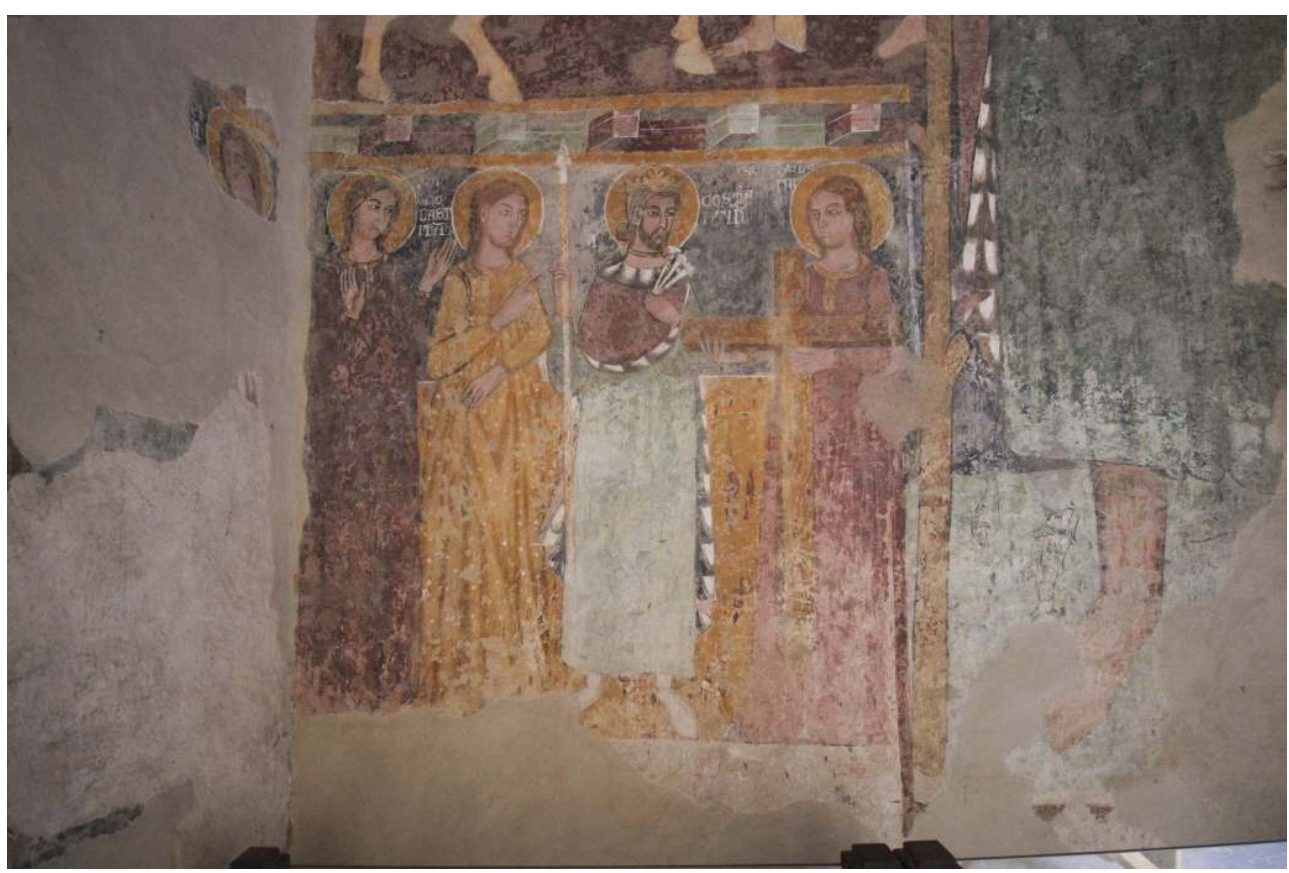

Alla munificenza del giudice Mariano IV, negli anni '60 del XIV secolo, sono con certezza attribuiti molti degli interventi di abbellimento della chiesa di Santa Chiara a Oristano, i cui dipinti recentemente riscoperti e restaurati non si esclude possano essere stati fatti in questo momento. ${ }^{62}$ Lo stesso Mariano interviene in altri cantieri importanti, come a San Pietro di Zuri (Ghilarza-Oristano), dove vengono realizzati dipinti murali oggi perduti, e a Sant'Antonio abate a Orosei (Nuoro), nella quale gli stemmi di famiglia sono ancora visibili nell'intonaco. ${ }^{63}$ Forse alla sua volontà possono essere ascritti anche gli interventi di abbellimento di un monumento meno noto, quale è la chiesa di San Lorenzo a Silanus (Nuoro) ${ }^{64}$. Le pareti nord e sud della chiesa ospitano dei lacerti pittorici che, pur noti alla critica, non erano stati oggetto di specifici studi. ${ }^{65}$ 
Datati da Renata Serra alla prima metà del XIII secolo, sarebbero viceversa da collocare alla metà del XIV secolo ${ }^{66} \mathrm{e}$ da legare al passaggio del possesso del distretto - o curatorìa - del Marghine al principe Mariano ${ }^{67}$.

17 In questo caso dunque si tratta di un territorio, quello del Marghine, che viene acquisito dal futuro Mariano IV e che rimane tra i suoi possedimenti. Come già detto, è evidente come molte delle campagne decorative del Trecento isolano vengano promosse all'interno di monumenti collocati in contesti territoriali di nuova acquisizione da parte del potere sovrano e signorile, quasi a voler marcare il territorio. I dipinti di Bosa sono realizzati quando il castello passa dalla famiglia signorile dei Malaspina in mano arborense, così come alcune delle pitture di Sant'Antonio abate di Orosei. Queste opere possono costituire, quindi, un segno visivo forte del cambiamento di dominio politico, testimonianza tangibile della azione di comunicazione del potere operata dal sovrano Mariano IV, la cui giovinezza passata a Barcellona, alla corte dei sovrani della Corona d'Aragona, costituisce un passaggio fondamentale per comprendere le modalità di rappresentazione del potere adottate alla corte d'Arborea a partire dal secondo quarto del Trecento. La decorazione ex novo di edifici frutto di acquisizioni territoriali, l'apposizione di stemmi dinastici, l'autorappresentazione all'interno del polittico di Ottana posso essere interpretati, oggi, come «mezzi d'effetto, idonei a comunicare gli elementi costituivi di quel potere, ma senza alcuna pretesa teorica di legittimazione» ${ }^{68}$.

Se i modelli "ideologici" possono essere iberici, rispetto all'esecuzione materiale delle opere si nota come il debito culturale con la penisola italiana rimane saldo lungo il corso del Trecento. Le maestranze che vengono chiamate a decorare le pareti degli edifici isolani sono relativamente aggiornate rispetto a ciò che accade nei più importanti cantieri centro-italici. Le opere su tavola ${ }^{69}$, seppur in quantità ridotta rispetto a contesti territoriali come la Toscana, arrivano anche nell'isola. Per alcune di queste si ha la certezza del legame ab antiquo, come nel caso del polittico di Ottana, per altre i riferimenti sono ancora tutti da cercare. Le fonti scritte suggeriscono inoltre la presenza, nell'Isola, anche di opere e di artisti provenienti dalla penisola iberica, dando vita a ottimi prodotti testimoni di una società dinamica e in pieno sviluppo. ${ }^{70}$ Applicando al giudicato d'Arborea le parole che Paola Vitolo utilizza per definire l'ambiente culturale della corte angioina di Napoli nel Trecento ${ }^{71}$, i giudici favoriscono l'arrivo di artisti nell'isola ${ }^{72}$, così come di manufatti di pregio, giungendo, almeno nel caso accertato del polittico di Ottana, a celebrare la propria persona e autorità, altresì creando dei modelli di autorappresentazione che troverebbero nei busti delle chiese di San Gavino Monreale e di Oristano due originalissimi esiti.

BIBLIOGRAFIA

BIBLIOGRAFIA PRIMARIA 
Armangué i Herrero - Cireddu Aste - Cuboni 2002 = J. Armangué i Herrero, A. Cireddu Aste, C. Cuboni (a cura di), Proceso contra los Arborea: archivio della Corona d'Aragona, Pisa, ETS, 2002, p. 262-288.

Conde y Delgado de Molina 2005 = R. Conde y Delgado de Molina, Diplomatario aragonés de Ugone II de Arborea, Sassari, Fondazione Banco di Sardegna, 2005.

Rubió i Lluch 1908 = A. Rubió i Lluch, Documents per a la Història de la cultura catalana mig-eval, Barcelona, Institut d'Estudis Catalans, 1908.

Zurita 1668 = J. Zurita, Anales de la Corona de Aragon, Zaragoza, Diego Dormer, 1668.

BIBLIOGRAFIA SECONDARIA

Baert 2013 = B. Baert, La leggenda della vera croce e la sua iconografia (VIII-XV secolo), s.v., in Enciclopedia Costantiniana, 2013, consultato il 15 dicembre 2018, http://www.treccani.it/ enciclopedia/la-leggenda-della-vera-croce-e-la-sua-iconografia-la-disseminazione-dei-ciclifigurativi-in-prospettiva-eur_\%28Enciclopedia-Costantiniana\%29/.

Bennert 1992 = U. Bennert, Art et propagande politique sous Philippe le Bel : le cycle des rois de France dans la Grande Salle du Palais de la Cité, in Revue de l'Art, 97, 1992, p. 46-58.

Biccone - Vecciu 2013 = L. Biccone, A. Vecciu, Bosa bizantina e giudicale. Nuove riflessioni sulla base dell'evidenza ceramica, in R. Martorelli (a cura di), Settecento Millecento. Storia, archeologia e arte nei "secoli bui" del Medioevo. Atti del Convegno di Studi, Cagliari, 2012, Cagliari, 2013, p. 341-364.

Brenk 1994 = B. Brenk, Committenza, s.v., in Enciclopedia dell'Arte Medievale, V, Roma, 1994, p. 203-218.

Brook et al. 1984 = L.L. Brook, F.C. Casula, M.M. Costa, A.M. Oliva, R. Pavoni, M. Tangheroni, Genealogie medioevali di Sardegna, Cagliari, 1984.

Cannas 1992 = M.C. Cannas, Alcuni aspetti della decorazione scultorea dell'ex-cattedrale di San Pantaleo in Dolianova: il busto del "giudice" d'Arborea Mariano II De Bas- Serra, in Medioevo. Saggi e Rassegne, 16, 1992, p. 220-221.

Cannas 2005 = M.C. Cannas, «Il re è nudo». Le effigi del giudice Barisone I d'Arborea re di Sardegna negli Annales Januenses di Oberto Cancellario, in L. Gallinari (a cura di), Genova una «porta» del Mediterraneo, Genova, 2005, p. 445-460.

Cardini 2015 = F. Cardini, I Francescani in Asia e la Custodia di Terrasanta, in A. Tartuferi, F. D'Arelli (a cura di), L'arte di Francesco. Capolavori d'Arte Italiana e terre d'Asia dal XIII al XV secolo. Catalogo della Mostra, Firenze, 2015, Firenze, 2015, p. 57-66.

Carta Raspi 1934 = R. Carta Raspi, Mariano IV conte del Goceano, visconte di Bas, giudice d'Arborea, Cagliari, 1934.

Casula 1984 = F.C. Casula, La scoperta dei busti in pietra dei re o giudici d'Arborea: Mariano IV, Ugone III, Eleonora con Brancaleone Doria, in Medioevo. Saggi e Rassegne, 4, 1984, p. 9-28.

Casula 1985 = F.C. Casula, La scoperta del Pantheon degli Arborea in Sardegna, in Nuova rivista storica, LXIX, I-II, 1985, p. 74-84.

Casula 1994 = F.C. Casula, La storia di Sardegna. Ristampa riveduta e corretta, Sassari, 1994.

Cingolani 2007 = S.M. Cingolani, La memòria dels reis. Les quatres grans cròniques, Barcelona, 2007.

Cioppi 2006 = A. Cioppi, Le grandi famiglie della Sardegna medievale: $i$ Malaspina, in Almanacco di Cagliari, Siena, 2006. 
Cioppi 2008 = A. Cioppi, Battaglie e protagonisti della Sardegna medievale, Cagliari, 2008.

Committenti 1992 = Committenti e produzione artistico-letteraria nell'alto Medioevo occidentale. Settimane di studio del Centro italiano di studi sull'alto Medioevo, Spoleto, 1991, Spoleto, 1992.

Coroneo 1988 = R. Coroneo, Per la conoscenza della scultura altomedioevale e romanica ad Oristano, in Biblioteca Francescana Sarda, II, 1988, p. 69-107.

Coroneo 1993 = R. Coroneo, Architettura romanica dalla metà del mille al primo ‘300, Nuoro, 1993.

Coroneo - Serra 2004 = R. Coroneo, R. Serra, Sardegna preromanica e romanica, Milano, 2004.

Corrao 1994 = P. Corrao, Celebrazione dinastica e costruzione del consenso nella Corona d'Aragona, in

P. Cammarosano (a cura di), Le forme della propaganda politica nel Due e nel Trecento, Roma, 1994, p. 133-156.

Delogu 1953 = R. Delogu, L'architettura del Medioevo in Sardegna, Roma, 1953.

García Marcilla 2000 = J.V. García Marcilla, Le immagini del potere e il potere delle immagini. I mezzi iconici al servizio della monarchia aragonese nel basso medioevo, in Rivista Storica Italiana, CXII, II, 2000, p. 569- 602.

Gardner 2015 = J. Gardner, Giotto e i francescani. Tre paradigmi di committenza, Roma, 2015.

Gimeno Blay 2006 = F.M. Gimeno Blay, Escribir, reinar. La experiencia gráfico-testual de Pedro IV el Ceremonioso, Madrid, 2006.

Golubovich 1918 = G. Golubovich, Peregrinationes Terrae Sanctae (saec. XIII), in Archivum Franciscanum Historicum, XI, 1918, p. 559-563.

Lucherini $2011=\mathrm{V}$. Lucherini, Le tombe angioine nel presbiterio di Santa Chiara a Napoli e la politica funeraria di Roberto d'Angiò, in A.C. Quintavalle (a cura di), Medioevo: i committenti. Atti del convegno internazionale, Parma, 2010, Milano, 2011, p. 477-504.

Lucherini $2015=\mathrm{V}$. Lucherini, Il potere medievale, la sua narrazione visuale e l'uso strumentale delle immagini, in Hortus Artium Medievalium, 21, 2015, p. 296-298.

Lusci 2014 = R. Lusci, Documenti sui giudici d'arborea nei protocolli di Bartomeu de Miramat e Pere Martí: Arxiu Històric de Protocols de Barcelona (1336-1362), in Aragón en la Edad Media, 25, 2014, p. 135-162.

Mattone 2008 = A. Mattone, Mariano d'Arborea, s.v., in DBI, consultato il 29 novembre 2018, http:// www.treccani.it/enciclopedia/mariano-d-arborea_(Dizionario-Biografico)/.

Meloni 1993 = G. Meloni (a cura di), Acta Curiarum Regnum Sardiniae. Il Parlamento di Pietro IV d'Aragona (1355), Cagliari, 1993.

Meloni 2002 = G. Meloni, L'Origine dei Giudicati, in M. Brigaglia,A. Mastino, G.G. Ortu (a cura di), Storia della Sardegna. 1. Dalla preistoria all'età bizantina, Roma-Bari, 2002, p. 1-32, 119.

Milanese 2016 = M. Milanese, Archeologia del castello di Bosa e archeologia urbana a Bosa, in A. Mattone, M.B. Cocco (a cura di), Bosa. La città e il suo territorio dall'età antica al mondo contemporaneo, Sassari, 2016, p. 298-317.

Molina 2003 = J. Molina, Gli artisti del re nel Trecento aragonese, in Annali della Scuola Normale Superiore di Pisa, 16, 2003, p. 193-213.

Molina Figueras 2013 = J. Molina Figueras, La memoria visual de una dinastía. Pedro IV El Ceremonioso y la retórica de las imágenes en la corona de Aragón (1336-1387), in Anales de Historia del Arte, 23, II, 2013, p. 219-241. 
Nuti 1992 = G. Nuti, Doria, Brancaleone, s.v., in DBI, consultato il 29 novembre 2018, http:// www.treccani.it/enciclopedia/brancaleonedoria_res-93ca6a32-87ec-11dc-8e9d-0016357eee51_(Dizionario-Biografico)/.

Oliva 1987 = A.M. Oliva, Il Goceano punto nevralgico della storia sarda. Medioevo: saggi e rassegne 12, p. $129-152$.

Oliva - Schena 2014 = A.M. Oliva, O. Schena (a cura di), Sardegna Catalana, Barcellona, 2014.

Ortu 2005 = G.G. Ortu, La Sardegna dei giudici, Nuoro, 2005.

Ortu 2017 = G.G. Ortu, La Sardegna tra Arborea e Aragona, Nuoro, 2017.

Pace 2000 = V. Pace, Arte a Roma nel Medioevo: committenza, ideologia e cultura figurativa in monumenti e libri, Napoli, 2000.

Pau 1994 = C. Pau, Un monastero nella storia della città. Santa Chiara di Oristano nei documenti dell'archivio. Parte Prima 1343-1699, in Biblioteca Francescana Sarda, V, 1994, p. 7-98.

Piras 2004 = A. Piras, La leggenda dell'Inventio Crucis e la sua rielaborazione nella Dottrina di Addai, in Theologica \& Historica, XIII, 2004, p. 305-315.

Quintavalle 2011 = A. C. Quintavalle (a cura di), Medioevo: $i$ committenti. Atti del convegno internazionale di studi, Parma, 2010, Milano, 2011.

Salvi 1991 = D. Salvi, Lo scavo nella chiesa di San Gavino, a San Gavino Monreale, in Quaderni Soprintendenza ai Beni Archeologici per le province di Cagliari e Oristano, VIII, 1991, p. 223-237.

Sanna 2008 = M.G. Sanna, Mariano d'Arborea, s.v., in DBI, consultato il 29 novembre 2018, http:// www.treccani.it/enciclopedia/mariano-d-arborea_res-61fad8d0e71d-11dd-804a-0016357eee51_(Dizionario-Biografico)/.

Schena 2015 = O. Schena, Pietro III d'Arborea, s.v., in DBI, consultato il 29 novembre 2018, http:// www.treccani.it/enciclopedia/pietro-iii-d-arborea_(Dizionario-Biografico).

Schena - Tognetti $2011=0$. Schena, S. Tognetti, La Sardegna medievale nel contesto italiano e mediterraneo (secc. XI-XV), Noceto, 2011.

Serra 1990 = R. Serra, Pittura e scultura dall'età romanica alla fine del ‘500, Nuoro, 1990.

Serreli 2003 = G. Serreli, L'Arborea: da Giudicato a Regno, in Archivio oristanese, 1, 2003, p. 43-52.

Serreli 2013 = G. Serreli, Il passaggio all'età giudicale: il caso di Càlari, in R. Martorelli (a cura di), Settecento Millecento. Storia, archeologia e arte nei "secoli bui" del Medioevo. Atti del Convegno di Studi, Cagliari, 2012, Cagliari, 2013, p. 63-81.

Silva Maroto 2013 = M.P. Silva Maroto, Donación Várez Fisa, Madrid, 2013.

Soddu 2005 = A. Soddu, I Malaspina e la Sardegna: documenti e testi dei secoli 12-14, Cagliari, 2005.

Soddu 2008 = A. Soddu, Forme di decentramento del potere nell'Arborea trecentesca: donnikellos, apanages e majoria de pane, in Bollettino di Studi Sardi, 1, 2008, p. 39-71.

Soddu 2014 = A. Soddu, Corona d'Aragona e Malaspina nella Sardegna del Trecento, in A.M. Oliva, O. Schena (a cura di), Sardegna catalana, Barcellona, 2014, p. 88-103.

Soddu 2016 = A. Soddu, La città di Bosa tra giudici di Torres e Malaspina (XII-XIII secolo), in A. Mattone, M.B. Cocco (a cura di), Bosa. La città e il suo territorio dall'età antica al mondo contemporaneo, Sassari, 2016, p. 288-297.

Soddu 2017 = A. Soddu, Signorie territoriali nella Sardegna medievale. I Malaspina (secc. XIII-XIV), Roma, 2017. 
Soddu c.s. = A. Soddu, La carta di popolamento del nuovo borgo di Goceano (1336), in E. Garau (a cura di), Il castello di Goceano, c.s.

Solmi 1917 = A. Solmi, Studi storici sulle istituzioni della Sardegna nel Medioevo, Cagliari, 1917.

Spiga 1992 = G. Spiga, Guida al Pantheon degli Arborea a san Gavino Monreale, Sassari, 1992.

Tasca 1986 = C. Tasca, Le influenze pisane nella produzione epigrafica sarda e catalana del XIV secolo, in Archivio Storico Sardo, 3, 1986, p. 62-80.

Tasca 1999 = C. Tasca, Titoli e privilegi dell'antica città di Bosa, Cagliari, 1999.

Tasca $2000=$ C. Tasca, La città di Bosa e i giudici di Arborea nel XIV secolo, in G. Mele (a cura di), Giudicato d'Arborea e Marchesato di Oristano: proiezioni mediterranee e aspetti di storia locale, Oristano, 2000, p. 1013-1043.

Tasca $2013=\mathrm{C}$. Tasca, Bosa nel tardo Medioevo. Fonti per lo studio di una città mediterranea, Cagliari, 2013.

Tosco 1997 = C. Tosco, Architetti e committenti nel romanico lombardo, Roma, 1997.

Tosco 2003 = C. Tosco, Il castello, la casa, la chiesa. Architettura e società nel medioevo, Torino, 2003.

Tosco 2009 = C. Tosco, La committenza vescovile nell' XI secolo nel romanico lombardo, in J. Jarnut, A. Köb, M. Wemhoff (a cura di), Bischöflisches Bauen im 11. Jahrhundert, Mittelalter Studien des Instituts zur Interdisziplinären Erfonrschung des Mittelalters-Paderborn, Monaco, 2009, p. 25-54.

Tosco 2012 = C. Tosco, Architettura e committenza nell'età di Leone IX, in G.M. Cantarella, A. Calzona (a cura di), La Reliquia del Sangue di Cristo. Mantova, l'Italia e l'Europa al tempo di Leone IX, Trento, 2012, p. 73-88.

Usai 2011 = N. Usai, Signori e chiese. Potere civile e architettura religiosa nella Sardegna giudicale (XI-XIV secolo), Cagliari, 2011.

Usai 2018 = N. Usai, La pittura nella Sardegna del Trecento, Perugia, 2018.

Usai c.s. = N. Usai, Pittura su tavola nella Corona d'Aragona tra XIV e XV secolo. Rapporti e relazioni tra Sardegna, penisola italiana e territori iberici, in V Ciclo di Studi Medievali. Atti del Convegno, Firenze, 2019, Arcore, c.s.

Usai - Nonne 2018 = N. Usai, C. Nonne, L'antica cattedrale di San Pantaleo a Dolianova (Cagliari), in IV Ciclo di Studi Medievali. Atti del Convegno di Studi, Firenze, 2018, Arcore, 2018, p. 497-504.

Vitolo 2014 = P. Vitolo, «Ecce rex vester». Christiformitas e spazio liturgico, in F. Aceto, S. D’Ovidio, E. Scirocco (a cura di), La chiesa e il convento di Santa Chiara. Committenza artistica, vita religiosa e progettualità politicanella Napoli di Roberto d'Angiò e Sancia di Maiorca, Battipaglia, 2014, p. 227-274.

Vitolo 2016 = P. Vitolo, Royauté et modèles culturels entre Naples, France et Europe. Les années de Robert

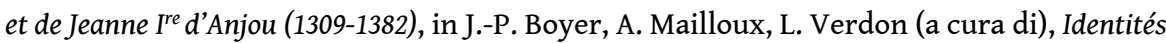
angevines entre Provence et Naples XIII ${ }^{e}-\mathrm{XV}^{e}$ siècle, Aix-en-Provence, 2016, p. 247-266.

Zedda - Pinna 2007 = C. Zedda, R. Pinna, La nascita dei giudicati. Proposta per lo scioglimento di un enigma storiografico, in Archivio storico e giuridico sardo di Sassari, n.s., 12, 2007, p. 27-118.

\section{NOTE}

1. Usai 2018. 
2. Relativamente ai quattro giudicati di rimanda a Solmi 1917; Brook et al. 1984; Casula 1994; Meloni 2002, p. 1-32; Ortu 2005; Zedda - Pinna 2007, p. 27-118; Serreli 2013, p. 63-81.

3. Sul potere signorile in Sardegna si veda, da ultimo, Soddu 2017.

4. Sulla Sardegna catalana, nei suoi molteplici aspetti si veda almeno Oliva - Schena 2014; Ortu 2017.

5. Nato probabilmente nel 1319, Mariano fu mandato a Barcellona con il fratello Giovanni dal padre nel 1323, per suggellare la rinnovata alleanza con la Corona. I due rampolli della famiglia giudicale arborense ebbero, alla corte catalana, un'educazione adeguata al loro rango, trattati come membri della famiglia regnante. Negli anni tra il 1331 e il 1332 il giovane Mariano ricevette dal padre Ugone i castelli di Goceano e Marmilla, provvedimento poi ratificato dal re catalano nel 1332 (Conde y Delgado de Molina 2005, p. 372-374, c. 308); tra il 1333 e il 1335 ebbe in feudo da Alfonso IV, insieme al fratello Giovanni, alcuni territori del Principato, tra cui il villaggio di Molins del Rey e il castello di Gelida. Mariano sposò nel 1336 la nobildonna catalana Timbors de Rocabertí e nello stesso momento fu armato cavaliere. Nel 1338 il donnikellu, o principe, Mariano fu designato conte del Goceano e signore della Marmilla e pochi anni dopo, forse nel 1341 o 1342, fece ritorno in Sardegna, dopo la lunga permanenza in terra iberica (Meloni 1993, p. 41-55).

6. Sulla figura di Mariano IV si faccia riferimento a Carta Raspi 1934; Oliva 1987; Serreli 2003, p. 43-52; Mattone 2008 con bibliografia precedente; Sanna 2008; Soddu 2008, p. 39-71; Lusci 2014, pp. 135-162; Soddu c.s., p. 15-16.

7. Lusci 2014, p. 135-136; Usai 2018, p. 345-348.

8. Lusci 2014, p. 140.

9. Lusci 2014, p. 144.

10. Lusci 2014, p. 145; Usai 2018, p. 345-349.

11. Il dipinto, una tempera su tavola su fondo oro, è diviso in tre scomparti principali: quello centrale raffigura i santi Nicola e Francesco. I due, al centro e a figura intera, sono inseriti in due nicchie archiacute, quasi due grandi monofore. La committenza è con chiarezza riconducibile alla volontà di Mariano, all'epoca principe e a quella del vescovo di Ottana Silvestro, entrambi raffigurati ai piedi del trono della Vergine nella cuspide del dipinto. Lo scomparto centrale ospita i santi Francesco e Nicola, ai lati otto storie della loro vita. Sull'opera si rimanda a Usai 2018, p. 170-187 con bibliografia precedente.

12. Mariano è vestito di un abito rosso, sulla sua spalla è poggiato un manto foderato di pelliccia di vaio, come è solito quando si tratti di personaggi di rango. Una lunga spada simbolo di regalità e attributo del cavaliere è attaccata alla vita tramite una cintura. Il volto del giovane, con una folta capigliatura bionda, incarna perfettamente il ritratto del nobile, cresciuto a Barcellona e ben consapevole di usi, tradizioni, consuetudini e istituzioni della corte catalana (Usai 2018, p. 170-182).

13. Zurita 1668, p. 466; Soddu c.s., p. 20.

14. Un sincero ringraziamento all'amico e collega Alessandro Soddu per i continui scambi di opinione sull'argomento e per i prezioni suggerimenti dati.

15. Si veda Usai 2018, p. 170-182 con bibliografia precedente. 
16. L'immagine è disponibile su https://www.museodelprado.es/coleccion/obra-dearte/virgen-de-tobed-con-los-donantes-enrique-ii-de/3977d582-961a-4fb9-8a4fea7d0573882b consultato il 19/11/2019.

17. Silva Maroto 2013, p. 16-19.

18. Usai 2018, p. 348.

19. Vitolo 2016, p. 248 con ulteriori indicazioni bibliografiche.

20. García Marsilla 2000, p. 571.

21. Molina Figueras 2013, p. 235.

22. García Marsilla 2000, p. 573.

23. Molina Figueras 2013, p. 220 con bibliografia precedente.

24. Corrao 1994, p. 133-156.

25. Molina Figueras 2013, p. 220.

26. Gimeno Blay 2006, p. 145-146.

27. Molina Figueras 2013, p. 221.

28. Molina 2003, p. 193-213.

29. Rubió i Lluch 1908.

30. L'ambiente oggi si presenta spoglio e privo di qualsivoglia ornamentazione. Risulta difficile ipotizzare quale fosse la distribuzione interna delle effigi, forse regolata da una seriazione cronologica dei regnanti, o sulla base di analogie tra i differenti personaggi. Rispetto ai modelli seguiti per la realizzazione di questo spazio l'ipotesi più plausibile pone un collegamento con la sala del Palais de la Cité a Parigi, decorata ai tempi di Filippo il Bello con una serie di sculture recenti le effigi dei monarchi (Bennert 1992, p. 46-58).

31. Molina Figueras 2013, p. 222.

32. Non solo in Sardegna il XIV secolo coincide con una fase di passaggio e di modificazioni politico-istituzionali. Gran parte dei regni dell'occidente mediterraneo vede negli anni centrali del Trecento dei cambi al vertice: in Francia i Capetingi lasciano lo scettro ai Valois, a Napoli (già dal 1266) arrivano gli Angiò in luogo degli Hoenstaufen, in Castiglia assurgono al vertice i Trastámara (Molinas Figueras 2013, p. 224). In questo contesto di cambiamenti celebrare l'antichità del lignaggio e la continuità dei governanti poteva costituire un modo di consolidare, almeno dal punto di vista del linguaggio visuale, la solidità del potere.

33. Casula 1985; Spiga 1992; Cannas 2005, p. 445-460.

34. L'edificio, pur ampiamente rimaneggiato, conserva evidenze della fase medievale sia nei reperti trovati a seguito dello scavo archeologico che ha interessato l'area, databili tra XIII e XIV secolo (Salvi 1991, p. 223-237), sia nelle iscrizioni che fissano il post e l'ante quem della fabbrica, collocabile tra 1347 e 1388. Il primo estremo cronologico è inciso in un concio, il secondo è in un'iscrizione dipinta nell'intonaco. Entrambi sono nel presbiterio, unica parte dell'edificio ancora visibile in forme medievali (Coroneo 1993, p. 283; Cannas 2005, p. 456-458).

35. Casula 1984, p. 9-28; Casula 1985, p. 74-85; Coroneo 1993, p. 283.

36. Per una sintesi biografica relativa ai personaggi citati si rimanda rispettivamente a Sanna 2008 (Mariano II); Schena 2015 (Pietro III e Costanza di Saluzzo); Mattone 2008 
(Mariano IV) disponibili su www.treccani.it/enciclopedia/(Dizionario-biografico) consultato il 29-11-2018.

37. Usai 2011, p. 84-85.

38. Vitolo 2016, p. 247.

39. Cingolani 2007, p. 2012.

40. Molina Figueras 2013, p. 223.

41. I giudici d'Arborea avevano stemmi sia statali sia personali. L'emblema dello stato era l'albero deradicato su campo argento. I membri della casata Bas- Serra, forse già nella seconda metà del XIII secolo (Casula 1984, p. 41), inseriscono un proprio vessillo aggiungendo all'insegna statale i pali d'Aragona, uno accanto all'altro. Mariano IV in un primo momento continua ad utilizzare l'insegna statale e lo stemma personale con i Pali d'Aragona affiancati, sovrapposti o al di sotto dell'Albero deradicato. Solo successivamente, con l'evolversi della situazione politica e l'incrinarsi dei rapporti con la corte catalana nel 1353, sostituirà lo stemma in uso con il solo albero deradicato (Casula 1984, p. 50-51).

42. Tasca 1986, p. 61; Coroneo 1988, p. 69-107.

43. Usai 2018 , p. 83 con bibliografia precedente.

44. Risulta più difficile condividere l'ipotesi di Maria Cristina Cannas che individua l'effige di Mariano II d'Arborea e di un vescovo nei due rilievi scultorei posti all'esterno del fianco settentrionale della chiesa di San Pantaleo di Dolianova (Sud Sardegna). L'edificio, edificato tra XII e XIII secolo, presenta una ricchissima decorazione scultorea d'apparato che oggi si presenta solo parzialmente leggibile a causa della consunzione della pietra. La figura in questione sembra presentare in effetti la corona sul capo e in mano un bastone, ma l'assenza di ulteriori elementi induce alla prudenza. Sulla chiesa si veda Coroneo - Serra 2004, p. 221-231; Usai 2018, p.61-80; Usai - Nonne 2018, p. 497-504. Sull'interpretazione dei rilievi si rimanda a Cannas 1992, p. 220-221; Cannas 2005, p. 452-453.

45. La chiesa, fondata o ripristinata a partire dal 1343 si presenta oggi, a causa di restauri novecenteschi, ad aula longitudinale, con due cappelle laterali per lato e presbiterio a pianta quadrangolare sormontato da volte a crociera costolonate. Analoga copertura, realizzata però in età moderna, si trova nell'aula. Una cantoria è addossata in controfacciata ed ospita alcune delle mensole lignee dell'originario tetto a capriate. La facciata è a capanna, sormontata da un piccolo campanile a vela al vertice (Usai 2018, p. 81-86).

46. Pau 1994, p. 27.

47. Pau 1994, p. 30-33.

48. Tasca 1986, p. 61; Coroneo 1988, p. 69-107; Usai 2018, p. 83.

49. Usai 2011, p. 77-99; Usai 2018, p. 85-86.

50. Vitolo 2016, p. 251.

51. Sul tema si vedano le posizioni di Lucherini 2011, p. 477-504; Vitolo 2014, p. 232-233.

52. Molina Figueras 2013, p. 233.

53. Il ciclo pittorico si stende lungo tre pareti (settentrionale, meridionale e occidentale) della chiesa di Nostra Signora de Sos Regnos Altos, all'interno del Castello di Serravalle (noto anche come Malaspina) a Bosa, in una serie di riquadri giustapposti uno all'altro. Il castello fino al 1349 è sede della signoria di Giovanni d'Arborea, fratello 
di Mariano e potentissimo feudatario nell'isola. Sull'argomento si vedano Tasca 1999; Tasca 2000, p. 1013-1043; Tasca 2013; Biccone - Vecciu 2013, p. 341-364; Milanese 2016, p. 301; Soddu 2016, p. 292. Sulla signoria malaspiniana in Sardegna si segnalano anche Soddu 2005; Cioppi 2006, s.n.p.; Soddu 2014b; Soddu 2017.

54. Tasca 2000, p. 1018-19.

55. Da ultimo si veda il recentissimo Usai 2018, p. 29-34 con ulteriori indicazioni bibliografiche.

56. Brenk, 1994, p. 203-18. Studi più specifici sui diversi aspetti sono in Committenti, 1992 e nel più recente Quintavalle ed., 2011. Tra i contributi dedicati a distinte aree geografiche o aspetti si ricordano, tra gli altri, Tosco, 1997; Pace, 2000; Tosco, 2003, p. 20-26; Tosco, 2009, p. 25-54; Tosco, 2012; Gardner, 2015.

57. Poli, 1999.

58. Golubovich 1918, p. 559-563; Cardini 2015, p. 57-66.

59. Piras 2004, p. 305-315; Baert 2013.

60. Ortu 2017.

61. Questa lettura è stata proposta per la prima volta in Usai 2018, p. 53-55 con ulteriori approfondimenti bibliografici.

62. Dell'intero ciclo pittorico che doveva decorare l'interno della chiesa residuano due riquadri, oggi nella seconda cappella a destra, di cui uno mostra una Madonna in trono con Bambino e figure ai lati, sulla sinistra, e una Crocifissione, sulla destra (Usai 2018, p. 88-93).

63. Per un'analisi dettagliata delle problematiche connesse ai due monumenti si rimanda al recentissimo lavoro monografico realizzato da chi scrive (Usai 2018, p. 81-132).

64. L'edificio, eretto in prossimità di una cava di calcare, è in pietra vulcanica, ha unica navata rettangolare con abside orientata e copertura con tetto ligneo (Delogu 1953, p. 140-141; Coroneo 1993, p. 154).

65. Serra 1990, p. 31.

66. Usai 2018, p. 132-135.

67. Nel 1331 il sovrano arborense Ugone II dona al figlio, il donnikellu Mariano, il castello di Goceano insieme alle curatorias di Dore, Anela, Marghine, Costavalle, Nuor (Soddu 2008, p. 48).

68. Lucherini 2015, p. 298.

69. Per un quadro approfondito dell'argomento si rimanda a Usai 2018, p. 138-218.

70. Usai 2018.

71. «Son contexte artistique ne se caractérise pas par la présence de personnalités influentes, susceptibles de fonder une tradition locale reconnaissable par des traits particuliers. Il se signale davantage par la capacité d'une classe dirigeante d'attirer des artistes de l'étranger, d'importer notamment de France et d'Italie centrale des produits prestigieux tels que manuscrits miniaturés et orfevreries, de revitaliser ainsi le milieu local, enfin de créer et de diffuser des propositions originales et des modèles d'autoreprésentation» (Vitolo 2016, p. 247).

72. È nota, solo per via documentaria, la presenza alla corte di Mariano IV del pittore di corte, tal "messer Morrone", non altrimenti noto (Armangué i Herrero - Cireddu Aste - 
Cuboni 2002, p. 262-288). Ben più celebre è il pittore del Cerimonioso, Ferrer Bassa, che realizzò diverse pale d'altare per i palazzi del re e i dipinti murali per la cappella di San Michele di Pedralbes, di fondazione reale (García Marsilla 2000, p. 598).

\section{RIASSUNTI}

Il tema delle rappresentazioni del potere e dell'uso delle immagini con scopi politici è oggetto oramai da diversi anni di un crescente numero di studi. Stemmi, oggetti di oreficeria, dipinti e sculture sono presi in esame non solo per il loro status di oggetti d'arte ma anche come prodotto della volontà di committenti facoltosi, spesso sovrani, che li utilizzano per accrescere il consenso intorno a loro. Il Trecento registra, in ambito mediterraneo, importanti personalità che utilizzano le immagini come forma di consolidamento visuale della loro autorità. Tra questi Roberto d'Angio, re di Napoli, e Pietro il Cerimonioso, sovrano della Corona d'Aragona, costituiscono dei casi - studio ampiamente esaminati. In questo saggio si vogliono offrire alcune riflessioni relative alla figura di Mariano IV, sovrano del giudicato d'Arborea, in rapporto ai governanti a lui coevi, in un momento storico, la metà del XIV secolo, nel quale la Sardegna sta entrando de facto nei territori della Corona d'Aragona.

The theme of representations of power and the use of images for political purposes has been the subject of a growing number of studies for several years now. Coats of arms, goldsmith's objects, paintings and sculptures are examined not only for their status as objects of art but also as a product of the will of wealthy, often sovereign, clients who use them to increase their consensus around them. The Mediterranean records important personalities who use images as a form of visual consolidation of their authority. Among these Robert of Anjou, king of Naples, and Peter IV the Ceremonious, ruler of the Crown of Aragon, construct widely examined case studies. In this essay we want to offer some reflections concerning the figure of Mariano IV, ruler of the giudicato of Arborea, in relation to the rulers coeval with him, in a historical moment, the midfourteenth century, in which Sardinia is entering de facto into the territories of the Crown of Aragon.

\section{INDICE}

Parole chiave : Committenti, Potere, Corona d'Aragona, Sardegna, Mariano IV d'Arborea

Keywords : Clients, power, Crown of Aragon, Sardinia, Mariano IV of Arborea

\section{AUTORE}

\section{NICOLETTA USAI}

Università degli Studi di Sassari - usai.nicoletta@gmail.com 